PROCEEDINGS OF THE

AMERICAN MATHEMATICAL SOCIETY

Volume 138, Number 4, April 2010, Pages 1439-1446

S 0002-9939(09)10196-X

Article electronically published on December 11, 2009

\title{
BEURLING'S PHENOMENON ON ANALYTIC HILBERT SPACES OVER THE COMPLEX PLANE
}

\author{
SHUYUN WEI
}

(Communicated by Nigel J. Kalton)

\begin{abstract}
In this paper, we show that Beurling's theorem on analytic Hilbert spaces over the complex plane analogous to the Hardy space or the Bergman space does not hold, but for finite co-dimensional quasi-invariant subspaces, they are generated by their wandering subspace if and only if they are generated by $z^{n}$ provided that the order of the reproducing kernels $K_{\lambda}(z)$ is less than 2 but not equal to 1 .
\end{abstract}

\section{INTRODUCTION}

Let $\operatorname{Hol}(\mathbb{C})$ denote the ring of all entire functions on the complex plane $\mathbb{C}$, and let $X$ be a Hilbert space contained in $\operatorname{Hol}(\mathbb{C})$. We call $X$ an analytic Hilbert space over $\mathbb{C}[\mathrm{CG}]$ if $X$ satisfies:

(a) $\left\{z^{n}\right\}_{n=0}^{\infty}$ forms an orthogonal basis for the Hilbert space $X$;

(b) the evaluation linear functional $E_{\lambda}(f)=f(\lambda)$ is continuous on $X$ for each $\lambda \in \mathbb{C}$.

Thus the reproducing kernels of $X$ have the form $K_{\lambda}(z)=\sum_{n=0}^{\infty} \frac{\bar{\lambda}^{n} z^{n}}{\left\|z^{n}\right\|^{2}}$.

The basic example of an analytic Hilbert space over the complex plane is the well known Fock space. Since the multiplication operator $M_{z}$ is unbounded in $X$, there is no nontrivial invariant subspace for $M_{z}$ in $X$ [GZh, $\mathrm{CGH}$. The concept of a quasi-invariant subspace was introduced in the Fock space GZh and in $X$ $\mathrm{CGH}$. We recall that a closed subspace $M$ of $X$ is quasi-invariant if the domain of $M_{z}$ is dense in $M$ and $z f \in X$ implies $z f \in M$ whenever $f \in M$. The structure of a quasi-invariant subspace of $X$ was studied in [GZh] and [CGH].

The classical Beurling Theorem $\mathrm{Beu}$ states that every invariant subspace $M \neq 0$ of the Hardy space has the form $M=[\phi]$ for some inner function $\phi$, where $[\phi]$ denotes the smallest invariant subspace containing $\phi$. An alternate statement is that $M$ is generated by its wandering subspace, i.e., $M=[M \ominus z M]$. Beurling's theorem has played an important role in operator theory and function theory. In Ric, Richter proved that the analogue of Beurling's theorem is true in the Dirichlet space. It is well known that the invariant subspace lattice of the Bergman space $L_{a}^{2}(\mathbb{D})$ is very complicated. In fact the dimension of $M \ominus z M$ can be an arbitrary positive integer or $\infty$ Hed2. Nevertheless, Aleman, Richter and Sundberg [ARS] obtained a Beurling-type theorem for the Bergman space. Moreover, Shimorin

Received by the editors May 6, 2009, and, in revised form, August 28, 2009.

2010 Mathematics Subject Classification. Primary 46E22, 47A15.

The author is partially supported by NNSFC in China, grant No. 10871140 . 
S1, S2 showed that the Beurling-type theorem is true for a large class of operators. In [HZ, by examining the zeros of an extremal function, Hedenmalm and Zhu discovered that the Beurling-type theorem fails in the weighted Bergman spaces with reproducing kernels $K_{\lambda}(z)=\frac{1}{(1-\lambda z)^{\alpha}}$ for $\alpha>5$. Chen and Hou studied the same problem for quasi-invariant subspaces of the Fock space [CH], they proved that if $M$ is a finite co-dimensional quasi-invariant subspace of the Fock space, then $[M \ominus z M]=M$ if and only if $M=\left[z^{n}\right]$ for some $n \geq 0$. Thus the Fock space is quite different from the Bergman space, the Hardy space and the Dirichlet space.

In this paper, we study the following problems:

1. Is there an analytic Hilbert space $Y$ such that the Beurling-type theorem holds in it?

2. Does the Beurling-type theorem in the Fock space hold in an arbitrary analytic Hilbert space $X$ ?

Let $M$ be a finite co-dimensional quasi-invariant subspace of $X$. We first give a description of $M^{\perp}$. Next we show that the Beurling-type theorem is not true in $X$, which is different from that in analytic Hilbert spaces over the unit disk. It turns out that it has a close connection to the order of reproducing kernels. We recall that an entire function $f(z)$ is called a function of finite order if

$$
\max _{|z|=r}|f(z)|<\exp \left(r^{k}\right)
$$

holds for sufficiently large values of $r$ and some $k>0$. The order of an entire function $f$ is the greatest lower bound of those values of $k$ for which the above inequality is fulfilled and is denoted by $\rho(f)$. We proved that if $\rho\left(K_{\lambda}(z)\right)<2$ and $\rho\left(K_{\lambda}(z)\right) \neq 1$, then $[M \ominus z M]=M$ if and only if $M=\left[z^{n}\right]$ for some $n \geq 0$. There are examples to show that the same result is not true if either $\rho\left(K_{\lambda}(z)\right)=1$ or $\rho\left(K_{\lambda}(z)\right) \geq 2$.

\section{Finite CO-DIMENSIONAL QUASI-INVARIANT SUBSPACES}

Let $M$ be a finite co-dimensional quasi-invariant subspace of $X$. Then there exists a polynomial $p$ such that $M=[p]$, where $[p]$ denotes the smallest quasiinvariant subspace containing $p[\mathrm{CGH}]$. In order to discuss Beurling's phenomenon, in this section we will study the structure of $M^{\perp}$ when $M$ is a finite co-dimensional quasi-invariant subspace of $X$, and we give a complete description of $M^{\perp}$. We note that the structure of $M^{\perp}$ may be well known to experts; see [Hed1 in the case of the Bergman space. We give its proof here just for convenience.

Theorem 2.1. Suppose that $p=z^{i_{0}}\left(z-\lambda_{1}\right)^{i_{1}} \cdots\left(z-\lambda_{m}\right)^{i_{m}}$ and $M=[p]$. Then

$$
\begin{array}{r}
M^{\perp}=\operatorname{span}\left\{1, z, \ldots, z^{i_{0}-1}, K_{\lambda_{1}}(z), z K_{\lambda_{1}}^{\prime}(z), \ldots, z^{i_{1}-1} K_{\lambda_{1}}^{\left(i_{1}-1\right)}(z),\right. \\
\left.\ldots, K_{\lambda_{m}}(z), z K_{\lambda_{m}}^{\prime}(z), \ldots, z^{i_{m}-1} K_{\lambda_{m}}^{\left(i_{m}-1\right)}(z)\right\} .
\end{array}
$$

Proof. We first show that $\left\{1, z, \ldots, z^{i_{0}-1}\right\} \subset M^{\perp}$. For each $f \in M$, there exists an entire function $f_{0}$, such that $f=z^{i_{0}} f_{0}$. So

$$
\left\langle f, z^{j}\right\rangle=\left\langle z^{i_{0}} f_{0}, z^{j}\right\rangle=0
$$

for $0 \leq j<i_{0}$ by the orthogonality of $\left\{z^{n}\right\}_{n=0}^{\infty}$. 
For each $f \in M$, there exists an entire function $f_{k}$, such that $f=\left(z-\lambda_{k}\right)^{i_{k}} f_{k}$, where $k=1, \ldots, m$. Thus

$$
\left\langle f, K_{\lambda_{k}}\right\rangle=\left\langle\left(z-\lambda_{k}\right)^{i_{k}} f_{k}, K_{\lambda_{k}}\right\rangle=\left(\lambda_{k}-\lambda_{k}\right)^{i_{k}} f_{k}\left(\lambda_{k}\right)=0 .
$$

Therefore $\left\{K_{\lambda_{1}}, \ldots, K_{\lambda_{m}}\right\} \subset M^{\perp}$.

Since $X$ is a reproducing Hilbert space with orthogonal basis $\left\{z^{n}\right\}_{n=0}^{\infty}$, its reproducing kernel $K_{\lambda}(z)$ has the form

$$
K_{\lambda}(z)=\sum_{n=0}^{\infty} \frac{z^{n} \bar{\lambda}^{n}}{\left\|z^{n}\right\|^{2}}
$$

Since $f(\lambda)=\left\langle f(z), K_{\lambda}(z)\right\rangle$, for $\lambda \neq 0$, we have

$$
\begin{aligned}
f^{\prime}(\lambda) & =\left\langle f(z), \frac{\partial K_{\lambda}(z)}{\partial \bar{\lambda}}\right\rangle \\
& =\left\langle f(z), \sum_{n=1}^{\infty} \frac{n z^{n} \bar{\lambda}^{n-1}}{\left\|z^{n}\right\|^{2}}\right\rangle \\
& =\frac{1}{\lambda}\left\langle f(z), z \sum_{n=1}^{\infty} \frac{n z^{n-1} \bar{\lambda}^{n}}{\left\|z^{n}\right\|^{2}}\right\rangle \\
& =\frac{1}{\lambda}\left\langle f(z), z K_{\lambda}^{\prime}(z)\right\rangle .
\end{aligned}
$$

Similarly, we get

$$
f^{(j)}(\lambda)=\frac{1}{\lambda^{j}}\left\langle f(z), z^{j} K_{\lambda}^{(j)}(z)\right\rangle
$$

If $1<k<i_{k}$, it is obvious that $f^{(k)}\left(\lambda_{i_{k}}\right)=0$ for all $f \in M$. Therefore,

$$
\left\langle f(z), z^{k} K_{\lambda_{i}}^{(k)}(z)\right\rangle=f^{(k)}\left(\lambda_{i}\right)=0 .
$$

Thus $z^{k} K_{\lambda_{i}}^{(k)}(z) \in M^{\perp}$. Note that both the codimension of $M$ and the number of elements in the right-hand side of $(*)$ are $i_{0}+i_{1}+\cdots+i_{k}$, and the elements in the right-hand side of $(*)$ are linearly independent. We have that $(*)$ holds, and this completes the proof.

\section{Beurling's Phenomenon ON $X$}

Let $M$ be a quasi-invariant subspace of the Fock space $F$ and $z M=\{z f \mid f \in$ $M, z f \in F\}$. Then $\operatorname{dim} M / z M$ can be any positive integer [HZh]. Chen and Hou proved that if $M$ is a finite co-dimensional quasi-invariant subspace of the Fock space, then $[M \ominus z M]=M$ if and only if $M=\left[z^{n}\right]$ for some $n \geq 0$.

In the present paper, we first examine whether the Beurling-type theorem in the Fock space is true for any finite co-dimensional quasi-invariant subspace of $X$. We have the following result:

Theorem 3.1. Suppose that $\rho\left(K_{\lambda}(z)\right)<2$ and $\rho\left(K_{\lambda}(z)\right) \neq 1$ for $\lambda \neq 0$ and let $p$ be a polynomial. Then $[p] \ominus[z p]$ generates $[p]$ if and only if $p=z^{n}$ for some $n \geq 0$.

Proof. Since the order of the entire function $f(z)=\sum_{n=0}^{\infty} c_{n} z^{n}$ is determined by the formula [Lev, p. 6]

$$
\rho(f)=\limsup _{n \rightarrow \infty} \frac{n \log n}{\log \left(1 /\left|c_{n}\right|\right)}
$$


we have

$$
\begin{aligned}
\rho\left(K_{\lambda}(z)\right) & =\limsup _{n \rightarrow \infty} \frac{n \log n}{\log \left(\left\|z^{n}\right\|^{2} /\left|\lambda^{n}\right|\right)} \\
& =\limsup _{n \rightarrow \infty} \frac{n \log n}{\log \left(\left\|z^{n}\right\|^{2}\right)} .
\end{aligned}
$$

So the order of $K_{\lambda}(z)$, which is completely determined by $\left\|z^{n}\right\|$, is independent of $\lambda$ for $\lambda \neq 0$.

If $p=z^{n}$, then $\left[\left[z^{n}\right] \ominus\left[z^{n+1}\right]\right]=\left[z^{n}\right]$ for $z^{n} \in\left[z^{n}\right] \ominus\left[z^{n+1}\right]$.

On the other hand, suppose that

$$
p(z)=z^{i_{0}-1}\left(z-\lambda_{1}\right)^{i_{1}} \cdots\left(z-\lambda_{m}\right)^{i_{m}} \quad \text { with } \prod_{i=1}^{m} i_{k} \neq 0 .
$$

Since $\operatorname{dim}[[p] \ominus[z p]]=1$, there exists $\phi \in[p] \ominus[z p]$ with $\phi \neq 0$. Using Theorem 2.1, we can write $\phi$ as

$$
\begin{aligned}
\phi(z) & =\sum_{n=0}^{i_{0}-1} d_{0 n} z^{n}+\sum_{n=0}^{i_{1}-1} d_{1 n} z^{n} K_{\lambda_{1}}^{(n)}(z)+\cdots+\sum_{n=0}^{i_{m}-1} d_{m n} z^{n} K_{\lambda_{m}}^{(n)}(z) \\
& =\sum_{n=0}^{\infty} b_{n} \frac{z^{n}}{\left\|z^{n}\right\|^{2}} .
\end{aligned}
$$

By the fact that $\rho\left(f^{\prime}\right)=\rho(f)$ and $\rho(f+g) \leq \max \{\rho(f), \rho(g)\}$, we get $\rho(\phi) \leq \rho\left(K_{\lambda}\right)$.

If $\rho(\phi)=0$, since $z^{k} K_{\lambda_{i}}^{(k)}(z)$ and $z^{j}$ are linearly independent, $\phi \neq c p$; thus $[[p] \ominus[z p]] \neq[p]$. So we assume next that $\rho(\phi) \neq 0$.

If $\liminf _{k \rightarrow \infty} b_{k} \neq 0$, then $\rho(\phi)=\rho\left(K_{\lambda}(z)\right) \neq 1$. Thus, by assumption, $\phi$ is an entire function with non-integer order. Therefore $Z(\phi)=\{z \mid \phi(z)=0\}$ is an infinite set $[$ Lev, p. 26] and $[p] \ominus[z p] \neq[p]$ because $p \notin[\phi]$.

If $\liminf _{k \rightarrow \infty} b_{k}=0$, say, $\lim _{n \rightarrow \infty} b_{k_{n}}=0$. Let

$$
\phi_{n}(z)=\sum_{k \neq k_{n}} b_{k} \frac{z^{k}}{\left\|z^{k}\right\|^{2}}+\sum_{m=1}^{n} b_{k_{m}} \frac{z^{k_{m}}}{\left\|z^{k_{m}}\right\|^{2}} \quad n=1,2, \ldots
$$

By Hurwitz's Theorem, $\phi(z)$ and $\phi_{n}(z)$ have the same number of zeros in any disk $D_{R}=\{z|| z \mid<R\}$ for large $n$. Suppose that $[[p] \ominus[z p]]=[p]$. Then we must have $\rho(\phi)=1$ and hence $\phi(z)=p(z) e^{C z}$. Thus $\phi(z)$ (and $\phi_{n}(z)$ for large $n$ ) has the same finite number of zeros as does $p(z)$. But this is impossible, since for each polynomial $q$ and each $B \neq 0$, if $q(z) e^{B z}=\sum_{k=0}^{\infty} c_{k} z^{k}$, then there exists $M>0$ such that $c_{k} \neq 0$ whenever $k>m$. This completes the proof.

In $\mathrm{CH}$, the authors proved that Theorem 3.1 holds in the Fock space. We note that the reproducing kernel of the Fock space is $e^{z \bar{\lambda}}$ and that $\rho\left(e^{z \bar{\lambda}}\right)=1$. So it is natural to ask whether the same result is true when $\rho\left(K_{\lambda}(z)\right)=1$. The following example gives a negative answer to the question.

Example 3.1. Let $X$ be a reproducing Hilbert space with reproducing kernels $K_{\lambda}(z)$ $=\sum_{n=1}^{\infty} \frac{2 n-1}{2^{n} n !} z^{n} \bar{\lambda}^{n}+1$. Then $[[z-1] \ominus[z(z-1)]]=[z-1]$.

Proof. It is easy to check that $\rho\left(\sum_{n=1}^{\infty} \frac{2 n-1}{2^{n} n !} z^{n} \bar{\lambda}^{n}+1\right)=1$. By Theorem 2.1, we have $[z(z-1)]^{\perp}=\operatorname{span}\left\{K_{1}(z), 1\right\}$. This together with $(z-1) e^{\frac{z}{2}}=K_{1}(z)-2$ 
implies that $(z-1) e^{\frac{z}{2}} \in[z-1] \ominus[z(z-1)]$. To complete the proof we only need to show that

or equivalently

$$
\left[(z-1) e^{\frac{z}{2}}\right]=[z-1]
$$

$$
\lim _{n \rightarrow \infty}\left\|(z-1) e^{\frac{z}{2}} S_{n}-(z-1)\right\|=0
$$

where $S_{n}=\sum_{k=0}^{n} \frac{z^{k}}{k ! 2^{k}}$.

In fact,

$$
\begin{aligned}
\left\|z^{k} e^{\frac{z}{2}}\right\|^{2} & =\sum_{n=0}^{\infty} \frac{\left\|z^{n+k}\right\|^{2}}{\left(2^{n} n !\right)^{2}} \\
& =\sum_{n=0}^{\infty} \frac{2^{n+k}(n+k) !}{2(n+k)-1} \cdot \frac{1}{\left(2^{n} n !\right)^{2}} \\
& \leq \sum_{n=0}^{\infty} k ! 2^{2 k} C_{n+k}^{k} \frac{1}{2^{n} n !}=\sum_{n=0}^{\infty} k ! 2^{2 k} \frac{2^{n+k}}{n ! 2^{n}} \\
& =\sum_{n=0}^{\infty} \frac{k ! 2^{3 k}}{n !}=k ! 2^{3 k} e .
\end{aligned}
$$

Thus

$$
\begin{aligned}
\left\|\sum_{k=m}^{n} \frac{z^{k} e^{\frac{z}{2}}}{k ! 2^{k}}\right\| & \leq \sum_{k=m}^{n} \frac{\left\|z^{k} e^{\frac{z}{2}}\right\|}{k ! 2^{k}} \\
& \leq \sum_{k=m}^{n} \frac{\sqrt{k ! 2^{2 k}}}{k ! 2^{k}}=\sum_{k=m}^{n} \frac{2^{k}}{\sqrt{k !}}
\end{aligned}
$$

Since $\sum_{k=0}^{\infty} \frac{2^{k}}{\sqrt{k !}}$ converges, both $z e^{\frac{z}{2}} S_{n}$ and $e^{\frac{z}{2}} S_{n}$ are Cauchy sequences in $X$. This together with the fact that $(z-1) e^{\frac{z}{2}} S_{n}$ converges point-wise to $z-1$ implies that $\lim _{n \rightarrow \infty}\left\|(z-1) e^{\frac{z}{2}} S_{n}-(z-1)\right\|=0$. This completes the proof.

In the case of $\rho\left(K_{\lambda}(z)\right) \geq 2$, Theorem 3.1 may fail. In fact we have the following example:

Example 3.2. For each $b \geq 2$, there exists an analytic Hilbert space $X$ with $\rho\left(K_{\lambda}(z)\right)=b$ such that $[(z-1)(z+1)] \ominus[z(z-1)(z+1)]=[(z-1)(z+1)]$ holds in $X$.

Proof. Let $b_{n}>0$ and $\lim _{n \rightarrow \infty} \frac{n \log n}{\log \left(1 /\left|b_{n}\right|\right)}=b \geq 2$, where $b$ may equal $+\infty$. Let

$$
K_{\lambda}(z)=\sum_{k=0}^{\infty} \frac{2 k+1}{2(k+1)} \frac{z^{2 k} \bar{\lambda}^{2 k}}{2^{k} k !}+\sum_{k=0}^{\infty} b_{2 k+1} z^{2 k+1} \bar{\lambda}^{2 k+1}
$$

Then $\rho\left(K_{\lambda}(z)\right)=b \geq 2$. In this case, we have

$$
(z+1)(z-1) e^{\frac{z^{2}}{2}}=\frac{1}{2} K_{1}(z)+\frac{1}{2} K_{-1}(z)-2 .
$$

By Theorem 2.1, we have

$$
(z+1)(z-1) e^{\frac{z^{2}}{2}} \in[(z-1)(z+1)] \ominus[z(z-1)(z+1)] .
$$


To complete the proof, we only need to show that

$$
\lim _{n \rightarrow \infty}\left\|\sum_{k=0}^{n} \frac{z^{2 k}}{2^{k} k !}(z-1)(z+1) e^{\frac{z^{2}}{2}}-(z-1)(z+1)\right\|=0 .
$$

In fact

$$
\begin{aligned}
\left\|z^{2 k} e^{\frac{z^{2}}{2}}\right\|^{2} & =\sum_{n=0}^{\infty} \frac{\left\|z^{2(n+k)}\right\|^{2}}{2^{2 n} n !^{2}} \\
& =\sum_{n=0}^{\infty} \frac{2^{n+k+1}(n+k+1) !}{[2(n+k)+1] 2^{2 n} n !^{2}} \\
& \leq \sum_{n=0}^{\infty} \frac{k ! 2^{k+1} C_{n+k+1}^{n}}{2^{n} n !} \\
& \leq 2^{2 k+2} k ! \sum_{n=0}^{\infty} \frac{1}{n !} \\
& =2^{2 k+2} k ! e .
\end{aligned}
$$

Thus

$$
\left\|z^{2 k} e^{\frac{z^{2}}{2}}\right\| \leq 2^{k+1} \sqrt{k ! e}
$$

Since

$$
\begin{aligned}
\left\|\sum_{k=0}^{n} \frac{z^{2 k}}{2^{k} k !} e^{\frac{z^{2}}{2}}\right\| & \leq \sum_{k=0}^{n}\left\|\frac{z^{2 k}}{2^{k} k !} e^{\frac{z^{2}}{2}}\right\| \\
& \leq \sum_{k=1}^{n} \frac{2 \sqrt{e}}{\sqrt{k !}}+\left\|e^{\frac{z^{2}}{2}}\right\|
\end{aligned}
$$

and $\sum_{k=1}^{n} \frac{2 \sqrt{e}}{\sqrt{k !}}<\infty$, we have that both $z^{2} \sum_{k=0}^{n} \frac{z^{2 k}}{k !} e^{z^{2}}$ and $\sum_{k=0}^{n} \frac{z^{2 k}}{k !} e^{z^{2}}$ are Cauchy sequences in $X$. Therefore

$$
\lim _{n \rightarrow \infty} \sum_{k=0}^{n} \frac{z^{2 k}}{2^{k} k !}(z-1)(z+1) e^{\frac{z^{2}}{2}}=(z-1)(z+1),
$$

and this completes the proof.

Example 3.1 shows that there exists an analytic Hilbert space over the complex plane such that the Beurling-type theorem may hold for a finite co-dimensional quasi-invariant subspace $M$ although $M \neq\left[z^{n}\right]$. However, the Beurling-type theorem does not hold for any analytic Hilbert space over the complex plane.

Theorem 3.2. Let $X$ be an analytic Hilbert space over the complex plane. Then there exists a quasi-invariant subspace $M$ of $X$ such that

$$
[M \ominus z M] \neq[M] .
$$

Proof. Since $X$ is an analytic Hilbert space over the complex plane, its reproducing kernel has the form

$$
K_{\lambda}(z)=\sum_{n=0}^{\infty} a_{n} z^{n} \bar{\lambda}^{n}=f(\bar{\lambda} z)
$$


where $a_{n}>0$ and $f(z)=K_{1}(z)=\sum_{n=0}^{\infty} a_{n} z^{n}$. Suppose $M=[z-1]$; if [[z1] $\ominus[z(z-1)]] \neq[z-1]$, we complete the proof. Otherwise, since $[z(z-1)]^{\perp}=$ $\operatorname{span}\left\{1, K_{1}(z)\right\}$, there exists an entire function $h(z)$ such that

$$
z(z-1) e^{h(z)}=K_{1}(z)+b_{1} .
$$

Thus

$$
K_{2}(z)=f(2 z)=K_{1}(2 z)=2 z(2 z-1) e^{h(2 z)}-b_{1} .
$$

On the other hand, if $[[z-2] \ominus[z(z-2)]]=[z-2]$, then there exists an entire function $r(z)$ such that

$$
z(z-2) e^{r(z)}=K_{2}(z)+b_{2}
$$

This implies that

$$
z(z-2) e^{r(z)}=2 z(2 z-1) e^{h(2 z)}-b_{1}+b_{2} .
$$

Letting $z=0$, we have that $-b_{1}+b_{2}=0$, which yields

$$
z(z-2) e^{r(z)}=2 z(2 z-1) e^{h(2 z)} .
$$

But this is impossible, and this completes the proof.

\section{REFERENCES}

[ARS] A. Aleman, S. Richter and C. Sundberg, Beurling's Theorem for the Bergman space, Acta Math., 177 (1996), 275-310. MR.1440934 (98a:46034)

[Beu] A. Beurling, On two problems concerning linear transformations in Hilbert space. Acta Math., 81 (1948), 239-255. MR0027954 (10:381e)

[CG] X. Chen and K. Guo, Analytic Hilbert Modules, Chapman \& Hall/CRC Res. Notes Math., 433, Chapman \& Hall/CRC, Boca Raton, FL, 2003. MR 1988884 (2004d:47024)

[CH] X. Chen and S. Hou, A Beurling-type theorem for the Fock space, Proc. Amer. Math. Soc. 131 (2003), no. 9, 2791-2795. MR:1974336 (2004c:46095)

[CGH] X. Chen, K. Guo and S. Hou, Analytic Hilbert spaces over the complex plane, J. Math. Anal. Appl. 268 (2002), 684-700. MR.1896222 (2003f:46038)

[Guo] K. Guo, Characteristic spaces and rigidity for analytic Hilbert modules, J. Funct. Anal. 163 (1999), 133-151. MR1682835 (2000b:46090)

[GZh] K. Guo and D. Zheng, Invariant subspaces, quasi-invariant subspaces, and Hankel operators, J. Funct. Anal. 187 (2001), 308-342. MR1875150 (2003b:47050)

[Hed1] H. Hedenmalm, A factorization theorem for square area-integrable analytic functions, J. Reine Angew. Math. 422 (1991), 45-68. MR.1133317 (93c:30053)

[Hed2] H. Hedenmalm, An invariant subspace of the Bergman space having the codimension two property, J. Reine Angew. Math. 443 (1993), 1-9. MR.1241125 (94k:30092)

[HZ] H. Hedenmalm and K. Zhu, On the failure of optimal factorization for certain weighted Bergman spaces, Complex Variables Theory Appl. 19 (1992), no. 3, 165-176. MR1284108 (95f:30064)

[HX] S. Hou and X. Xu, Notes on ordered reproducing Hilbert spaces over the complex plane, J. Math. Anal. Appl. 317 (2006), 448-455. MR2208931 (2007e:46022)

[HZh] S. Hou and Z. Zheng, Zero-based subspaces and quasi-invariant subspaces of the Bargmann-Fock space, preprint, 2008.

[Lev] B. Ya. Levin, Lectures on entire functions, Translations of Mathematical Monographs, 150, Amer. Math. Soc., Providence, RI, 1996. MR1400006 (97j:30001)

[Ric] S. Richter, Invariant subspaces of the Dirichlet shift. J. Reine Angew. Math. 386 (1988), 205-220. MR936999 (89e:47048) 
[S1] S. M. Shimorin, Wold-type decompositions and wandering subspaces for operators close to isometries, J. Reine Angew. Math. 531 (2001), 147-189. MR.1810120 (2002c:47018)

[S2] S. M. Shimorin, On Beurling-type theorems in weighted $l^{2}$ and Bergman spaces, Proc. Amer. Math. Soc. 131 (2003), 1777-1787. MR.1955265 (2004a:47008)

Department of Mathematics, Suzhou University, Jiangsu Suzhou, 215006, People's Republic of China

E-mail address: swei@suda.edu.cn 